\title{
Csf3r mutations in mice confer a strong clonal HSC advantage via activation of Stat5
}

\author{
Fulu Liu, ${ }^{1}$ Ghada Kunter, ${ }^{1}$ Maxwell M. Krem, ${ }^{1}$ William C. Eades, ${ }^{1}$ Jennifer A. Cain, ${ }^{1}$ \\ Michael H. Tomasson, ${ }^{1}$ Lothar Hennighausen, ${ }^{2}$ and Daniel C. Link'
}

1Department of Medicine, Division of Oncology, Washington University School of Medicine, Saint Louis, Missouri, USA.

'Laboratory of Genetics and Physiology, NIDDK, NIH, Bethesda, Maryland, USA.

\begin{abstract}
A fundamental property of leukemic stem cells is clonal dominance of the bone marrow microenvironment. Truncation mutations of CSF3R, which encodes the G-CSF receptor (G-CSFR), are implicated in leukemic progression in patients with severe congenital neutropenia. Here we show that expression of a truncated mutant Csf $3 r$ in mice confers a strong clonal advantage at the HSC level that is dependent upon exogenous G-CSF. G-CSF-induced proliferation, phosphorylation of Stat5, and transcription of Stat 5 target genes were increased in HSCs isolated from mice expressing the mutant Csf $3 r$. Conversely, the proliferative advantage conferred by the mutant Csf $3 r$ was abrogated in myeloid progenitors lacking both Stat5A and Stat5B, and HSC function was reduced in mice expressing a truncated mutant $C s f 3 r$ engineered to have impaired Stat 5 activation. These data indicate that in mice, inappropriate Stat 5 activation plays a key role in establishing clonal dominance by stem cells expressing mutant $C s f 3 r$.
\end{abstract}

\section{Introduction}

There is accumulating evidence suggesting that most leukemia is initiated by rare cancer stem cells, referred to as leukemic stem cells (LSCs). LSCs are functionally defined as cells able to generate leukemia upon transfer to an appropriate host (1). Two fundamental and interrelated properties of LSCs are the capacity for self-renewal and clonal dominance. Clonal dominance refers to the clonal expansion of LSCs within the bone marrow microenvironment at the expense of normal hematopoietic cells.

The mechanisms by which mutations and/or epigenetic changes that accumulate during leukemogenesis contribute to clonal dominance of LSCs are largely unknown. On a functional level, acquisition of clonal dominance represents a competitive advantage in the bone marrow microenvironment, as suggested by stochastic models (2). A requisite property for clonal dominance is the ability to self-renew. The capacity for self-renewal can be achieved either by transformation of HSCs with inherent self-renewal capacity or by the acquisition of this ability in committed progenitor populations during leukemogenesis (3). The molecular pathways that confer clonal dominance have been largely unexplored, though a recent study utilizing retroviral mutagenesis showed that the majority of retroviral integrations associated with clonal dominance (both malignant and nonmalignant) occurred near genes involved in the regulation of HSC self-renewal, proliferation, or apoptosis (4).

Severe congenital neutropenia (SCN) is a congenital bone marrow failure syndrome characterized by severe neutropenia present from birth, an arrest of myeloid differentiation at the promyelocyte/myelocyte stage, and a marked propensity to develop acute myeloid leukemia (AML) or a myelodysplastic syndrome (MDS) (5, 6). The cumulative risk of MDS/AML in patients with SCN after

Nonstandard abbreviations used: AML, acute myeloid leukemia; G-CSFR, G-CSF receptor; KSL, c-kit ${ }^{+} \mathrm{Sca}^{+}$lineage ${ }^{-}$, LSC, leukemic stem cell; MDS, myelodysplastic syndrome; SCN, severe congenital neutropenia.

Conflict of interest: The authors have declared that no conflict of interest exists. Citation for this article: J. Clin. Invest. 118:946-955 (2008). doi:10.1172/JCI32704.
10 years is $11 \%-21 \%(7,8)$. Treatment with G-CSF is effective in increasing neutrophil counts and preventing infections (9); however, the quantity and duration of G-CSF treatment are correlated with an increased risk of $\operatorname{MDS} / \operatorname{AML}(7,8)$.

Nonsense mutations of CSF3R that truncate the distal cytoplasmic portion of the G-CSF receptor (G-CSFR) are present in approximately $40 \%$ of patients with SCN (10). These mutations are acquired and strongly associated with the development of AML/MDS (10). Expression of mutant G-CSFR in myeloid cell lines results in enhanced proliferative and survival signals (11-13). Moreover, transgenic mice carrying truncating mutations of Csf $3 r$ display a hyperproliferative response to G-CSF $(14,15)$. However, these mice do not develop MDS/AML; thus, a causal relationship between CSF3R mutations and leukemia has not been established.

In the present study, we investigated the mechanisms by which cells containing mutant CSF3R gain clonal dominance in vivo. Surprisingly, competitive repopulation studies using HSC from targeted transgenic (knockin) mice carrying the Csf $3 r$ truncation mutation showed no clonal advantage. However, short-term treatment with G-CSF resulted in a marked competitive advantage of mutant HSC. We utilized this inducible model to characterize the molecular pathways mediating HSC clonal dominance. These studies suggest that accentuated Stat5 activation plays a key role in establishing clonal dominance by HSC-expressing mutant Csf3R.

\section{Results}

The d715 G-CSFR confers a competitive advantage at the HSC level in a $G$-CSF-dependent fashion. We previously described the generation of mice carrying a targeted (knockin) mutation of their Csf $3 r$ gene that is representative of the truncation mutations found in SCN (15). Of note, the targeted transgenic (knockin) approach preserves the genomic organization of the Csf3r allele; accordingly, the mutant G-CSFR is expressed in a development- and lineage-specific fashion. These mice, termed "d715 G-CSFR," have normal basal granulopoiesis but exhibit an exaggerated neutrophil response to exogenous G-CSF. To examine the effect of the d715 G-CSFR on HSC function, a competitive repopulation assay was performed. 
A

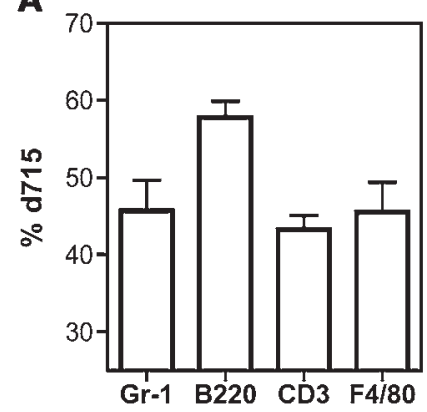

B

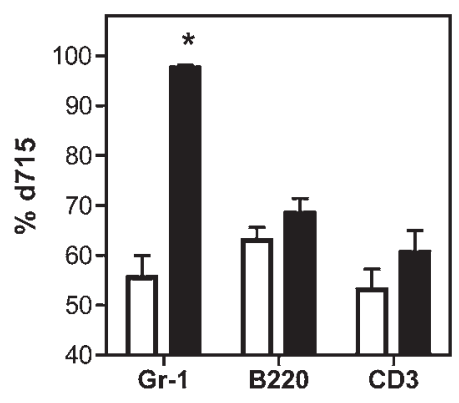

C



Figure 1

The d715 G-CSFR confers a competitive repopulation advantage that is dependent upon exogenous G-CSF administration. Irradiated syngeneic mice were reconstituted with a 1:1 ratio of d715 G-CSFR $($ Ly5.2+ $)$ and wild-type $($ Ly5.1+) bone marrow cells. (A) The percentage of blood neutrophils (Gr-1), B lymphocytes (B220), T lymphocytes (CD3), and monocytes (F4/80) that were derived from d715 G-CSFR (d715) cells is shown $(n=9)$. (B and C) The mice were then treated with G-CSF $(10 \mu \mathrm{g} / \mathrm{kg} / \mathrm{d} ; n=5)$ or saline alone $(n=4)$ for 21 days and the contributions of d715 G-CSFR cells in the blood (B) and bone marrow (C) were measured. Data represent the mean \pm SEM. ${ }^{*} P<0.05$.

Recipient mice were reconstituted with a 1:1 ratio of $\mathrm{d} 715 \mathrm{G}-\mathrm{CSFR}$ and wild-type bone marrow cells, and the contribution of $\mathrm{d} 715$ G-CSFR cells to hematopoiesis was determined by flow cytometry. At 6 months after transplantation, the contribution of $\mathrm{d} 715$ G-CSFR cells to the neutrophil, B lymphocyte, T lymphocyte, and monocyte lineages in the blood was near the predicted 50\% level, indicating that the $\mathrm{d} 715 \mathrm{G}-\mathrm{CSFR}$ does not confer a competitive advantage (Figure 1A).

In patients with $\mathrm{SCN}$, the systemic level of G-CSF is high, either because of increased endogenous production or its pharmacologic administration (16). To simulate this increase in systemic G-CSF, we treated the chimeric mice with G-CSF $(10 \mu \mathrm{g} / \mathrm{kg} / \mathrm{d})$ for 3 weeks and repeated the chimerism analysis (Figure 1, B and C). After G-CSF treatment, nearly all of the circulating neutrophils were derived from d715 G-CSFR cells (saline, $55.5 \% \pm 4.4 \%$ versus G-CSF, $97.6 \% \pm 1.2 \% ; P<0.001)$. Likewise, nearly all $(98.6 \% \pm 0.7 \%)$ of $\mathrm{Gr}-1^{+}$granulocytic cells in the bone marrow were of $\mathrm{d} 715$ G-CSFR origin after G-CSF treatment. A significant increase in the contribution of $\mathrm{d} 715 \mathrm{G}-\mathrm{CSFR}$ cells to the B lymphocyte lineage in the bone marrow also was observed (saline, $61.2 \% \pm 6.4 \%$ versus G-CSF, $89.1 \% \pm 5.7 \%$; $P<0.001$ ); the lack of significant increase in circulating B or T lymphocytes after only 3 weeks of G-CSF treatment likely reflects the long half-life of these cells.

The increase in $\mathrm{d} 715 \mathrm{G}-\mathrm{CSFR}$ chimerism in the B lymphocyte lineage raised the possibility that the $\mathrm{d} 715 \mathrm{G}$-CSFR confers a competitive advantage at the HSC level after G-CSF treatment. To address this possibility, chimerism in c-kit ${ }^{+} \mathrm{Sca}^{+}$lineage ${ }^{-}$(KSL) cells, a cell population enriched in HSCs, was assessed. In saline-treated mice, the percentage of KSL cells derived from d715 G-CSFR cells was $53.3 \% \pm 11.5 \%$ (Figure 2). After 3 weeks of G-CSF treatment, this percentage increased to $97.8 \% \pm 0.8 \%(P<0.001)$. We next asked whether short-term (3-week) treatment with G-CSF results in durable increases in d715 G-CSFR chimerism. A separate cohort of chimeric mice was generated by transplanting equal numbers of wild-type and d715 G-CSFR bone marrow cells into recipient mice. Five months after transplantation, these mice were treated for 3 weeks with G-CSF or saline and then followed for an additional 3 months. As expected, immediately after G-CSF treatment, virtu-
A

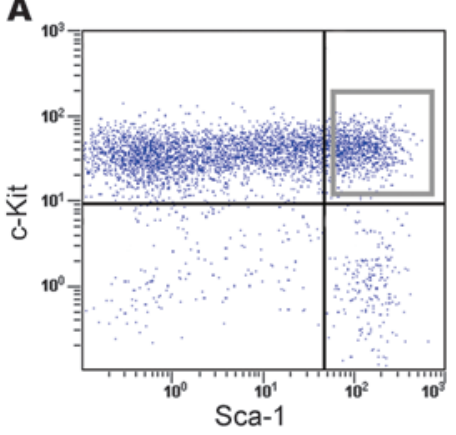

B

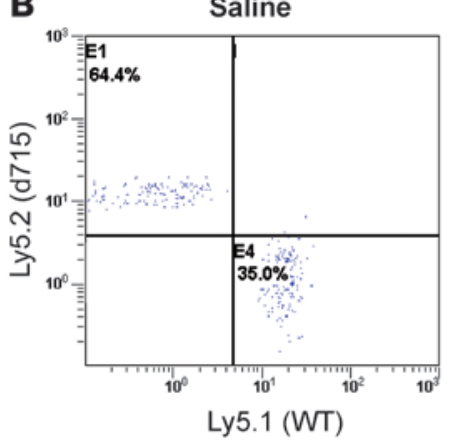

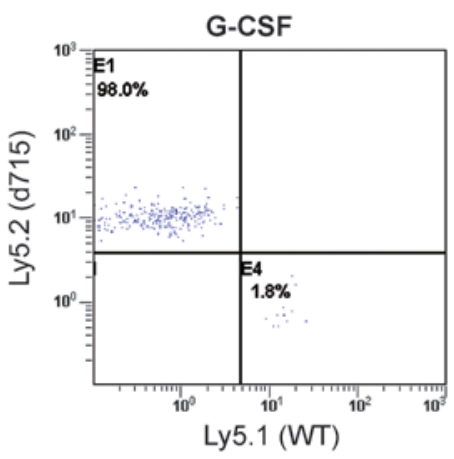

C

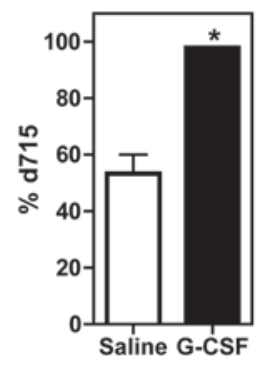

Figure 2

The contribution of d715 G-CSFR cells to the KSL cell compartment is increased after G-CSF treatment. Chimeric mice generated as described in Figure 1 were treated with saline or G-CSF $(10 \mu \mathrm{g} / \mathrm{kg} / \mathrm{d})$ for 21 days, and the percentage of KSL cells in the bone marrow derived from d715 G-CSFR cells was determined. (A) Representative scatter plot showing the strategy to identify KSL cells (boxed); data are gated on lineage- cells (data not shown). (B) Representative scatter plots gated on KSL cells showing the percentage of d715 G-CSFR (Ly5.2+) cells. (C) The percentage of KSL cells derived from d715 G-CSFR cells is shown $\left(n=4-5\right.$ each). Data represent the mean \pm SEM. ${ }^{*} P<0.001$. 
A

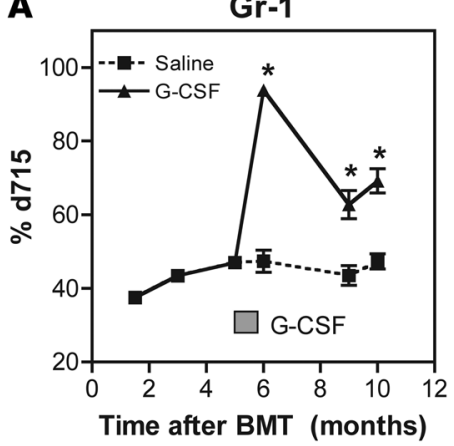

B

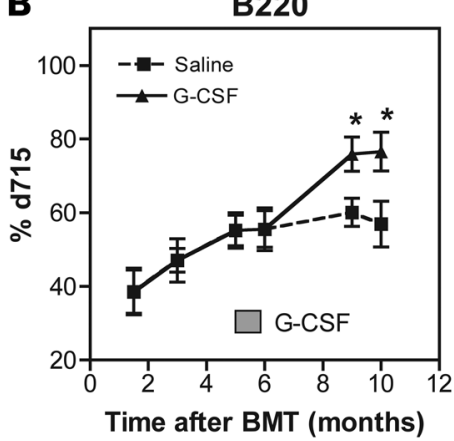

C

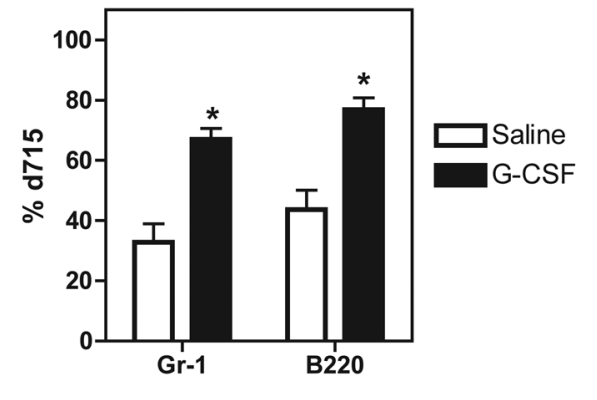

Figure 3

Long-term d715 G-CSFR chimerism following G-CSF treatment is increased. Chimeric mice generated as described in Figure 1 were treated 5 months after bone marrow transplantation (BMT) with saline alone or G-CSF (10 $\mu \mathrm{g} / \mathrm{kg} / \mathrm{d}$ ) for 21 days (indicated by the gray box). The percentage of circulating neutrophils (A) and B lymphocytes (B) derived from d715 G-CSFR cells was determined at the indicated times $(n=6-7$ for each group). (C). Bone marrow was harvested from chimeric mice after treatment with G-CSF or saline and transplanted into secondary recipients ( $n=5-10$ each cohort of recipient mice). Shown is the percentage of d715 G-CSFR cells in the blood of the secondary recipients 4 months after transplantation. Data represent the mean \pm SEM. ${ }^{*} P<0.001$.

ally all of the circulating neutrophils were of d715 G-CSFR origin (Figure $3 \mathrm{~A}$ ). This increase persisted 3 months after discontinuing G-CSF treatment; the percentage of circulating neutrophils derived from d715 G-CSFR cells was $69.2 \% \pm 3.28 \%$ versus $47.3 \% \pm 2.07 \%$ for saline-treated mice $(P<0.001)$. Strikingly, G-CSF treatment also significantly increased the contribution of $\mathrm{d} 715 \mathrm{G}$-CSFR cells to the B lymphocyte lineage. Three months after discontinuing G-CSF, the percentage of circulating B lymphocytes derived from d715 G-CSFR cells was $76.6 \% \pm 1.98 \%$ versus $56.9 \% \pm 2.35 \%$ for saline-treated mice $(P<0.001)$.

A defining feature of HSC is their ability to support multilineage long-term engraftment after transplantation into recipient mice. Consequently, we transplanted bone marrow from chimeric mice after treatment with G-CSF or saline into secondary recipient mice, and d715 G-CSFR chimerism was assessed 4 months after transplantation. A significant increase in $\mathrm{d} 715 \mathrm{G}$-CSFR contribution to circulating neutrophils $(66.9 \% \pm 3.68 \%$ versus $32.8 \% \pm 6.09 \%$; $P<0.001)$ and B lymphocytes $(76.8 \% \pm 3.90 \%$ versus $43.7 \% \pm 6.36 \%$; $P<0.001)$ was detected in secondary recipients that had received G-CSF treated bone marrow (Figure 3C). Collectively, these data show that the d715 G-CSFR confers a strong competitive advantage to HSC in a G-CSF-dependent fashion.

G-CSF-induced proliferation is increased in 2715 G-CSFR HSCs. The clonal advantage of $\mathrm{d} 715 \mathrm{G}$-CSFR HSCs suggested that proliferation, survival, and/or self-renewal were enhanced. To characterize the effect on proliferation, wild-type or d715 G-CSFR mice were treated with a single dose of G-CSF, and 24 hours later, the cell cycle status of KSL cells in the bone marrow was measured (Figure 4). At baseline, a similar percentage of KSL were in the $\mathrm{S} / \mathrm{G}_{2} / \mathrm{M}$ phases of the cell cycle (wild type, $8.9 \% \pm 2.0 \%$ versus $\mathrm{d} 715$ G-CSFR, $10.0 \% \pm 3.0 \% ; P=\mathrm{NS}$ ). After treatment with G-CSF, the percentage of cycling KSL cells increased significantly in d715 G-CSFR mice $(34.4 \% \pm 2.4 \%)$, with a trend to increased cycling in wild-type KSL cells $(20.0 \% \pm 3.8 \%)$. Of note, G-CSF induced a significantly greater proliferative response in d715 G-CSFR compared with wild-type KSL cells $(P<0.05)$.

The KSL cell population is heterogeneous, containing both HSCs and lineage-committed progenitors. Consequently, we repeated these experiments using more restrictive criteria to identify HSCs.
Specifically, we determined the fraction of CD $150^{+} \mathrm{CD} 48^{-} \mathrm{CD} 41^{-}$ lineage ${ }^{-}$cells that labeled with BrdU in vivo after G-CSF treatment. A previous study showed that 1 in 2.2 of these cells has long-term repopulating activity (17). Consistent with this study, the frequency of CD $150^{+} \mathrm{CD} 48^{-} \mathrm{CD} 41^{-}$lineage ${ }^{-}$cells in the bone marrow of wild-type mice was approximately $0.005 \%$ (Figure 4C). A similar frequency was observed in $\mathrm{d} 715 \mathrm{G}-\mathrm{CSFR}$ mice and did not change with G-CSF treatment (data not shown). In wild-type mice, G-CSF treatment had no effect on BrdU incorporation of CD $150^{+} \mathrm{CD} 48^{-}$ CD41-lineage- cells (Figure 4D). In contrast, in d715 G-CSFR mice, G-CSF treatment significantly stimulated BrdU incorporation in these cells (saline, $15.4 \% \pm 7.6 \%$ versus G-CSF, $42.8 \% \pm 12.9 \%$; $P=0.004)$. Together, these data suggest that proliferative signaling in HSC is greatly enhanced by the d715 G-CSFR.

Short-term G-CSF treatment induces the activation of Stat 3 and/or Stat5 target genes in KSL cells. To elucidate the molecular mechanisms by which the d715 G-CSFR confers a clonal HSC advantage, RNA expression profiling experiments were performed. Specifically, wild-type or d715 G-CSFR mice were treated with a single dose of G-CSF or saline, and 3 hours later KSL cells were sorted from the bone marrow by flow cytometry. The 3-hour time point was chosen to identify early transcriptional targets of G-CSF signaling in KSL cells. Of note, this brief exposure to G-CSF had no discernible effect on KSL number or flow cytometry characteristics (data not shown). Data from 3 independent experiments were pooled; genes that were consistently upregulated or downregulated by G-CSF treatment are summarized in Supplemental Tables 1 and 2 (supplemental material available online with this article; doi:10.1172/JCI32704DS1). Two criteria were used to identify genes differentially regulated by G-CSF in d715 G-CSFR KSL cells. First, genes were selected in which the magnitude of the change in mRNA expression after G-CSF treatment in d715 G-CSFR KSL cells was at least 2.0-fold greater than that observed with wild-type KSL cells. Second, a significant difference in the absolute gene chip signal between G-CSF-treated wild-type and d715 G-CSFR KSL cells was required. Based on these criteria, 14 genes were identified (Table 1). Strikingly, there is evidence that Stat3 and/or Stat 5 regulate the mRNA expression of 6 of these genes, including Sprr2a (18), Torg (19, 20), Pim2 (21), Cdkn1a (22, 23), 

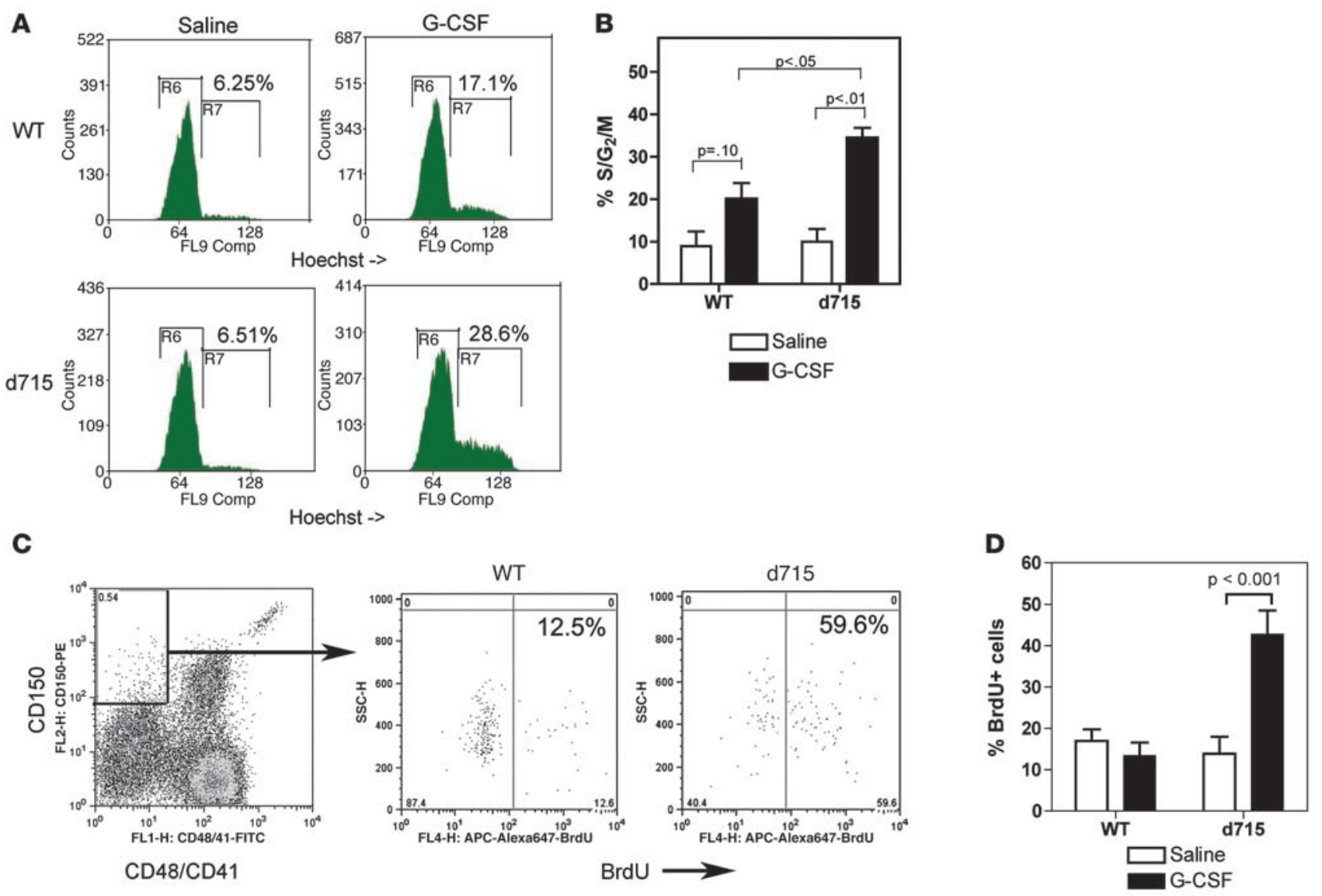

C

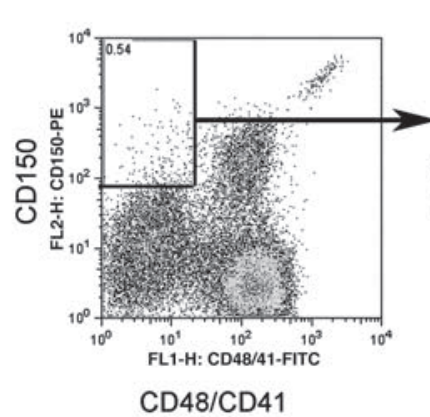

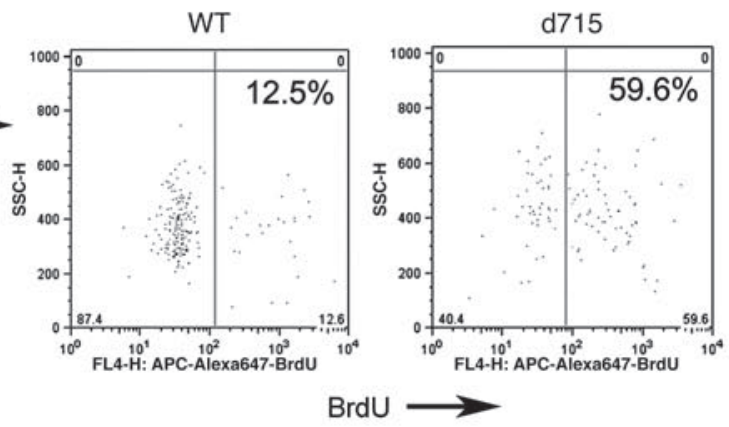

Figure 4

G-CSF-induced proliferation of d715 G-CSFR HSCs is increased. Wild-type or d715 G-CSFR mice were treated with saline or G-CSF (2 doses of $100 \mu \mathrm{g} / \mathrm{kg}, 12$ hours apart); in some cases (C and D), mice also received BrdU (2 doses of $1 \mathrm{mg}, 12$ hours apart). (A) Representative histograms showing Hoechst staining of KSL cells. (B). Shown is the percentage of $\mathrm{KSL}$ cells in the $\mathrm{S} / \mathrm{G}_{2} / \mathrm{M}$ phases of the cell cycle $(n=3-4$ per condition). (C) Representative scatter plots showing the gating strategy and BrdU staining of CD150+CD48-CD41-lineage- cells. (D) Shown is the percentage of $\mathrm{CD} 150^{+} \mathrm{CD} 48^{-} \mathrm{CD} 41^{- \text {lineage }}{ }^{-}$cells that labeled with $\mathrm{BrdU}(n=5-6$ per condition). Data represent the mean $\pm \mathrm{SEM}$.

Cish (24), and Socs2 (25). These data suggest the hypothesis that accentuated activation of Stat 3 and/or Stat 5 mediates the HSC clonal advantage of the d715 G-CSFR.

Stat 5 but not Stat 3 activation by G-CSF is accentuated in 7715 G-CSFR $K S L$ cells. A flow cytometric assay to quantify the phosphorylation of Stat 3 and Stat 5 was used to assess the activation of these proteins in KSL cells (26). We first validated the assay in primary murine myeloid cells (Figure 5, A and B). Consistent with previous studies utilizing gel shift assays $(12,27)$, Stat 5 activation by the d715 G-CSFR in primary myeloid cells was markedly enhanced (AUC: wild type, $148 \pm 28$ and d715 G-CSFR, $371 \pm 86 ; P<0.05$ ). Again, consistent with previous reports $(12,27)$, Stat 3 activation in d715 G-CSFR myeloid cells, though delayed, was comparable with wild-type cells (AUC: wild type, 2,053 \pm 546 and d715 G-CSFR, 2,711 $\pm 815 ; P=\mathrm{NS})$.

Similar experiments were performed with KSL cells (Figure 5, $\mathrm{C}$ and D). Compared with wild-type cells, both the magnitude and duration of Stat5 activation by G-CSF was increased in d715 G-CSFR cells (AUC: wild type, $203 \pm 73$ and d715 G-CSFR, $771 \pm 187 ; P=0.05)$. In contrast, Stat 3 activation by G-CSF in d715 G-CSFR KSL cells was significantly reduced at early time points after G-CSF stimulation (Figure 5D), with a trend to a decrease in overall Stat 3 activation (AUC: wild type, 1,315 \pm 491 and d715 G-CSFR, 1,020 $\pm 530 ; P=0.16)$. These data show that Stat 5 activation by the $\mathrm{d} 715$ G-CSFR in KSL cells was enhanced, whereas Stat 3 activation was attenuated.

The hyperproliferative response of the 7715 G-CSFR is abrogated in Stat5deficient myeloid progenitors. We next sought to assess the effect of the loss of Stat 5 on the clonal dominance conferred by the d715 G-CSFR. However, the engraftment potential of Stat $5 A^{-/-} \times$Stat $5 B^{-/-}$ HSC is markedly impaired $(28,29)$, precluding the direct assessment of the d715 G-CSFR on HSC function in Stat5-deficient cells. Instead, we determined whether the previously described hyperproliferative phenotype conferred by the d715 G-CSFR on myeloid progenitors is abrogated by Stat 5 deficiency. In brief, hematopoietic cells harvested from the fetal liver of wild-type or Stat $5 A^{-1-} \times$ Stat $5 B^{-/-}$mice were transduced with retrovirus expressing wild-type or $\mathrm{d} 715 \mathrm{G}$-CSFR and colony formation in response to G-CSF measured. As reported previously, expression of the $\mathrm{d} 715$ G-CSFR in Stat5-sufficient cells resulted in a significant increase in both colony number and average colony size (Figure 6, A and B). In Stat $5 \mathrm{~A}^{-/-} \times$Stat $5 \mathrm{~B}^{-/-}$cells, G-CSF-induced colony formation was reduced, and the hyperproliferative response conferred by the d715 G-CSFR was abrogated. 
Table 1

RNA expression profiling

\begin{tabular}{|c|c|c|c|c|c|c|c|c|}
\hline \multirow[b]{2}{*}{ Gene } & \multicolumn{3}{|c|}{ WT } & \multicolumn{3}{|c|}{ d715 G-CSFR } & \multirow[b]{2}{*}{ Ratio $^{A}$} & \multirow[b]{2}{*}{$P$ value ${ }^{\mathrm{B}}$} \\
\hline & Saline & G-CSF & Fold change & Saline & G-CSF & Fold change & & \\
\hline Tcrg & $318 \pm 91$ & $202 \pm 88$ & 0.64 & $266 \pm 24$ & $2,492 \pm 438$ & 9.37 & 14.7 & $<0.005$ \\
\hline Sprr2a & $810 \pm 124$ & $2,098 \pm 903$ & 2.59 & $599 \pm 122$ & $21,072 \pm 6,628$ & 35.2 & 13.6 & $<0.05$ \\
\hline Tnfsf11 & $435 \pm 116$ & $251 \pm 95$ & 0.58 & $232 \pm 108$ & $1,116 \pm 74$ & 4.81 & 8.34 & $<0.005$ \\
\hline Cish & $2,730 \pm 525$ & $3,005 \pm 110$ & 1.10 & $2,829 \pm 801$ & $13,794 \pm 556$ & 4.88 & 4.43 & $<0.0005$ \\
\hline Pim2 & $1,382 \pm 293$ & $1,981 \pm 530$ & 1.43 & $1,040 \pm 326$ & $6,337 \pm 1,523$ & 60.09 & 4.25 & $<0.05$ \\
\hline Enah & $230 \pm 83$ & $1,495 \pm 127$ & 6.50 & $628 \pm 203$ & $17,320 \pm 2,265$ & 27.6 & 4.24 & $<0.005$ \\
\hline Zfpn1a4 & $167 \pm 68$ & $181 \pm 68$ & 10.08 & $248 \pm 92$ & $1,105 \pm 76$ & 4.46 & 4.11 & $<0.0005$ \\
\hline Socs2 & $903 \pm 62$ & $1,315 \pm 335$ & 1.46 & $2,210 \pm 868$ & $11,020 \pm 1,488$ & 4.99 & 3.42 & $<0.005$ \\
\hline Cdkn1a & $987 \pm 171$ & $1,541 \pm 206$ & 1.56 & $791 \pm 135$ & $4,057 \pm 568$ & 5.13 & 3.29 & $<0.01$ \\
\hline Serpina3g & $22,519 \pm 5,363$ & $31,260 \pm 4,761$ & 1.39 & $20,302 \pm 5,034$ & $83,742 \pm 13,422$ & 4.12 & 2.97 & $<0.05$ \\
\hline Bcat1 & $137 \pm 49$ & $572 \pm 95$ & 4.18 & $75 \pm 34$ & $883 \pm 46$ & 11.8 & 2.82 & $<0.05$ \\
\hline Cacnb2 & $769 \pm 769$ & $1,621 \pm 77$ & 2.11 & $585 \pm 277$ & $3,018 \pm 595$ & 5.16 & 2.45 & $<0.05$ \\
\hline$L t b 4 r 1$ & $1,025 \pm 259$ & $730 \pm 198$ & 0.71 & $3,231 \pm 2,069$ & $276 \pm 34$ & 0.09 & 0.12 & $<0.05$ \\
\hline Bhlhb2 & $127 \pm 52$ & $1,267 \pm 179$ & 9.98 & $723 \pm 583$ & $2,501 \pm 133$ & 3.46 & 0.35 & $<0.005$ \\
\hline
\end{tabular}

The mean \pm SEM raw signal of experiments performed in triplicate is shown. ARatio of the fold change after G-CSF treatment between wild-type and d715 G-CSFR cells. ${ }^{B}$ Comparing G-CSF-treated wild-type KSL cells with G-CSF-treated d715 G-CSFR KSL cells.

Loss of Stat 3 and Stat 5 activation by the d715F G-CSFR is associated with decreased HSC function. To further test the hypothesis that Stat3 and/or Stat5 activation mediates the clonal HSC advantage of the d715 G-CSFR, we next studied d715F G-CSFR-targeted transgenic (knockin) mice. In the d715F G-CSFR mutant, the sole remaining tyrosine (Y704) of d715 G-CSFR is mutated to phenylalanine. We previously showed that Stat 3 and Stat 5 activation by the $\mathrm{d} 715 \mathrm{~F}$ G-CSFR in primary murine myeloid cells is markedly impaired (27). Similar results were obtained with KSL cells (Figure 7, A and B). In fact, no detectable Stat 5 or Stat 3 activation was observed in d715F KSL cells following G-CSF stimulation.

To determine whether the loss of Stat 3 and Stat 5 activation by the $\mathrm{d} 715 \mathrm{~F}$ G-CSFR affected HSC function, we repeated the competitive repopulation assay using a 1:1 ratio of $\mathrm{d} 715 \mathrm{~F}$ G-CSFR and wild-type bone marrow cells. Consistent with the known defect in granulopoiesis in $\mathrm{d} 715 \mathrm{~F}$ G-CSFR mice (27), the contribution of $\mathrm{d} 715 \mathrm{~F}$ G-CSFR cells to the neutrophil lineage was consistently less than that observed in the B or T lymphocyte lineages (Figure 7, $\mathrm{C}-\mathrm{E})$. Chimeric mice were treated with G-CSF for 3 weeks starting 4 months after transplantation. Despite the loss of Stat 3 and Stat5 activation, a significant increase in d715F G-CSFR chimerism in the neutrophil lineage was observed. However, this increase was transitory, returning to control levels after stopping the G-CSF. In fact, a gradual decrease in $\mathrm{d} 715 \mathrm{~F}$ G-CSFR contribution to neutrophils, B lymphocytes, and T lymphocytes was observed over time, irrespective of whether G-CSF was given. These data suggest that the $\mathrm{d} 715 \mathrm{~F}$ G-CSFR confers a competitive disadvantage to HSC.

\section{Discussion}

Truncation mutations of CSF3R are strongly and uniquely associated with the development of MDS/AML in patients with SCN. Whereas truncation mutations of CSF3R are present in $78 \%$ of cases of MDS/AML in patients with SCN (10), they are observed in only $1 \%-2 \%$ of de novo AML cases $(10,30,31)$. These mutations are acquired and achieve clonal dominance in the human bone marrow microenvironment. In the present study, we show that expression of a representative G-CSFR truncation mutant confers a strong clonal advantage at the HSC level. However, the clonal dominance is dependent upon exogenous G-CSF administration; similar results were observed after treating mice for 3 weeks with 2 different doses of G-CSF: $10 \mu \mathrm{g} / \mathrm{kg} / \mathrm{d}$ and $100 \mu \mathrm{g} / \mathrm{kg} / \mathrm{d}$ (data not shown). The requirement for high systemic levels of G-CSF may explain the unique association of CSF3R mutations with SCN, since in SCN, systemic levels of G-CSF are high due to increased endogenous production or pharmacologic administration (16). A novel feature of this model is the inducibility of the HSC clonal dominance, providing a tool to explore molecular mechanisms for this process.

There is increasing evidence that HSC quiescence is associated with long-term repopulating activity. Transplantation studies of phenotypically defined HSC fractionated based on cell cycle have shown that the quiescent fraction has the highest repopulating activity $(32,33)$. Moreover, loss-of-function mutations for Cdkn1a $\left(\mathrm{p} 21^{\text {waf1 }}\right)(34)$ or Pten $(35,36)$ that result in excessive HSC proliferation are associated with loss of long-term repopulating ability. In contrast, in the present study, we show that mutant Csf3r confers increased repopulating activity despite inducing greater proliferation of HSC (as defined by KSL or CD $150^{+} \mathrm{CD} 41^{-} \mathrm{CD} 48^{-}$lineage ${ }^{-}$ cells). These observations suggest the possibility that the nature of the proliferative signals generated by mutant Csf $3 r$ may preserve the long-term repopulating activity of HSC. In this regard, it is interesting to note that Cdkn1a ( $\mathrm{p} 21^{\text {waf }}$ ) expression is strongly induced by G-CSF in Csf3r mutant HSC (Table 1). As noted above, $C d k n 1 a$ is a negative regulator of HSC proliferation and helps maintain HSC quiescence. Thus it is possible that simultaneous induction of proliferative signals and Cdkn1a expression may prevent the loss of long-term repopulating activity associated with uncontrolled HSC proliferation.

There is considerable evidence linking Stat 5 to the regulation of HSC function. Stat 5 is activated by several cytokine receptors implicated in the regulation of HSC, including MPL and FLT3 $(37,38)$. Moreover, loss of function mutations for both Stat5A and Stat5B lead to a profound defect in hematopoietic repopulating activity $(28,29,39)$. Conversely, expression of a constitutively active form of Stat 5 in both murine and human hematopoietic progenitors promoted the self-renewal and expansion of multi- 
A

pSTAT5 in Gr-1+ cells

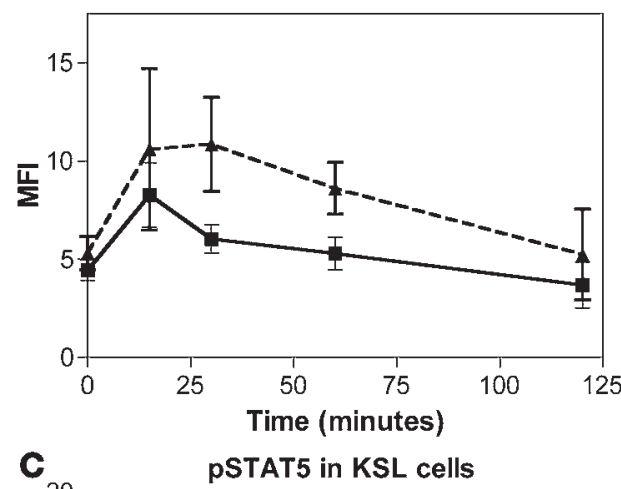

B

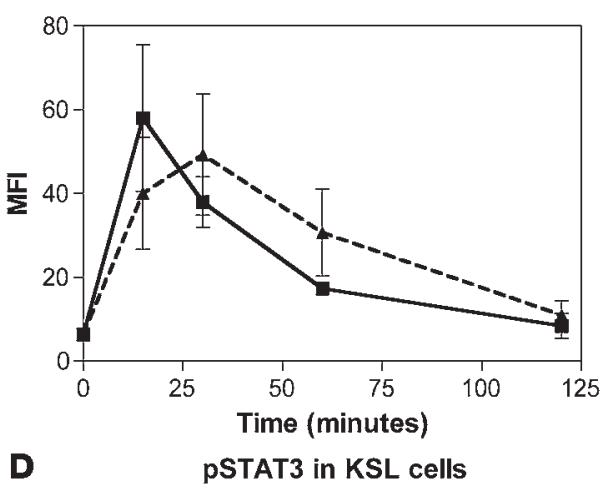

D

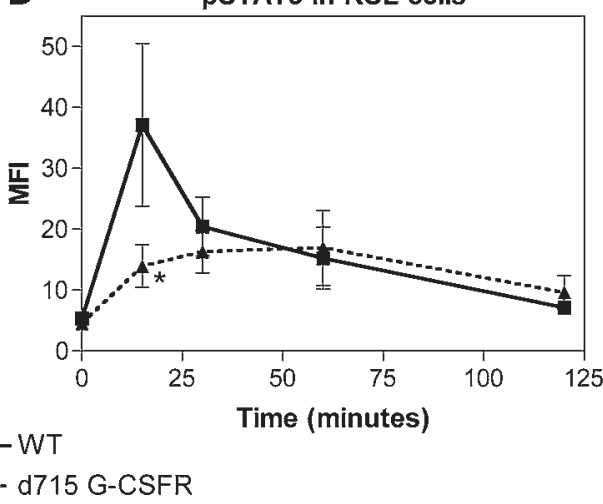

\section{Figure 5}

Stat5 but not Stat3 activation by G-CSF is enhanced in d715 G-CSFR cells. Wild-type or d715 G-CSFR bone marrow cells were treated in vitro for the indicated times with G-CSF $(100 \mathrm{ng} / \mathrm{ml})$, and the cells were then incubated with antibodies specific for phosphorylated Stat5 or Stat3. (A and B) The MFI of Gr-1+ (myeloid) cells for pStat5 (A) and pStat3 (B) is shown. (C and $\mathbf{D})$ The MFI of KSL cells for pStat5 (C) and pStat3 (D) is shown. Data represent the mean \pm SEM ( $n=4$ per cohort). ${ }^{*} P<0.05$ compared with wild-type cells at the same time point.

potential progenitors ex vivo $(37,40)$. Herein, we show that Stat5 but not Stat3 phosphorylation by G-CSF is accentuated in Csf $3 R$ mutant KSL cells. Accordingly, several well-known Stat5 target genes, including PIM2 and Cdkn1a, are induced to a greater degree by G-CSF in Csf $3 R$ mutant compared with wild-type KSL cells. Moreover, though the severe repopulating defect of Stat $5 \mathrm{~A}^{-/-} \times$ Stat $5 \mathrm{~B}^{-/-}$cells precluded a direct measure of HSC activity, we show that the proliferative advantage of myeloid progenitors expressing mutant (d715) Csf $3 R$ is dependent upon Stat5. Finally, to directly assess the importance of Stat 5 activation by G-CSF in the regulation of HSC function, we characterized the long-term repopulating activity of HSCs expressing a mutant (d715F) Csf $3 R$ engineered to have impaired Stat 5 activation. In fact, the competitive repopulating activity of $\mathrm{d} 715 \mathrm{~F}$ HSCs was reduced compared with wild-type HSCs. A caveat of this experiment is that other signaling pathways besides Stat 5 (and Stat 3 ) may be disrupted by the d715F mutation. Collectively, these data suggest that accentuated Stat 5 activation by mutant Csf $3 r$ mediates the clonal HSC advantage.

Stat 5 has been implicated in leukemogenesis. Constitutive Stat 5 activation has been detected in approximately $25 \%$ of leukemias (41), and it is specifically associated with leukemias carrying internal tandem duplications of FLT3 (FLT3 ITD) (42). Expression of constitutively active Stat 5 in hematopoietic cells results in a rapidly fatal myeloproliferative disease in mice $(37,41,43)$. Studies shown). Studies are underway to see whether any of these genes are activated at later time points after G-CSF stimulation. Of the genes differentially regulated by G-CSF in Csf3r mutant KSL cells (Table 1), several have been implicated in the regulation of HSC function or leukemogenesis. As already discussed, Cdkn1a has been shown to regulate HSC self-renewal (34). Pim2 is a serine-threonine kinase that has been shown to play a pivotal role in the transmission of proliferative and survival signals by FLT3 ITD $(47,57)$. The importance of these genes is currently being investigated through the use of the relevant loss-of-function transgenic mice.

The high rate of leukemic transformation in SCN, and the dismal prognosis once MDS or AML develops, has led to considerable debate over the timing of allogeneic HSC transplantation (HSCT) in patients with SCN. Unfortunately, there are no randomized clinical trials comparing HSCT with standard therapy in SCN. However, based on its strong association with MDS/AML, the presence of CSF3R mutations has been proposed as an indication for HSCT, even in the absence of MDS/AML (10). The present study supports this approach. Though none of the Csf3r mice developed MDS/ AML, our data showing that the mutant Csf3r confers a clonal HSC advantage increases the likelihood that CSF3R mutations contribute to leukemic transformation. Alternatively, the dependence of the HSC clonal advantage on high systemic levels of G-CSF suggests that discontinuing G-CSF administration may result in the 

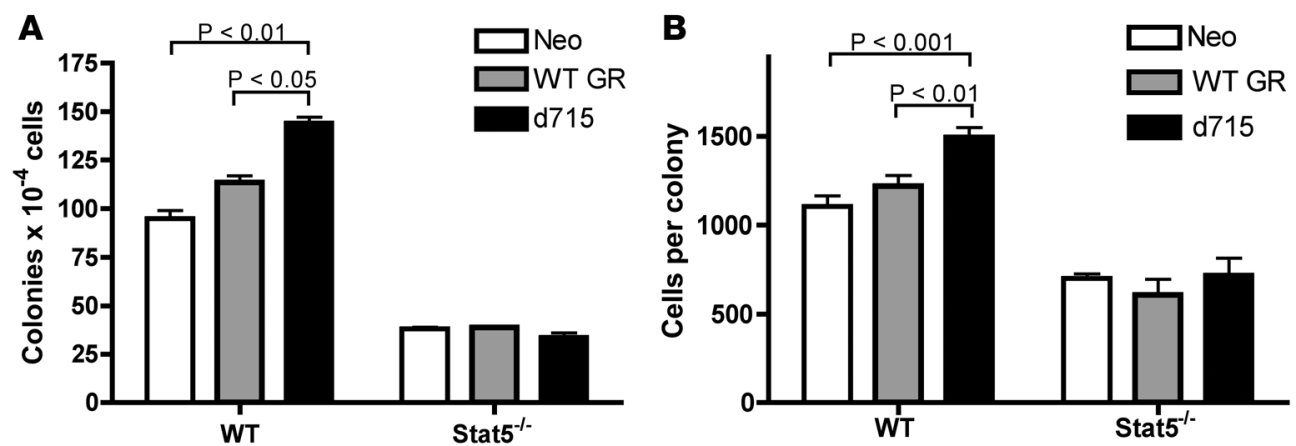

Figure 6

Stat5 deficiency abrogates the hyperproliferative response of the d715 G-CSFR in myeloid progenitors. E12-E15 fetal liver cells from wildtype or Stat5 $\mathrm{A}^{-/-} \times \mathrm{Stat}_{5 \mathrm{~B}} \mathrm{~B}^{-/-}\left(\mathrm{Stat5}^{-/-}\right)$mice were transduced with retrovirus expressing neomycin phosphotransferase alone (Neo) or this gene and either wild-type G-CSFR (WT GR) or d715 G-CSFR. Cells were cultured in $10 \mathrm{ng} / \mathrm{ml}$ of G-CSF and geneticin to select for transduced cells. Shown is the number of colonies $(\mathbf{A})$ and average colony size (B) observed after 7-10 days. Similar results were observed in cultures stimulated with $1 \mathrm{ng} / \mathrm{ml}$ of G-CSF (data not shown). No colonies formed in the absence of G-CSF (data not shown). Data represent the mean \pm SEM of 3 independent experiments.

loss (or at least stabilization) of the CSF3R mutant clone. Indeed, there is a case report in which a patient with a CSF3R mutation and AML had a spontaneous remission of AML after stopping G-CSF (58). However, treatment with G-CSF is proven to improve surviv- al in patients with SCN, and there currently is no other effective therapy to increase neutrophil counts in patients with SCN. In the absence of such a therapy, it may be prudent to consider allogeneic HSCT in all patients with SCN who acquire a CSF3R mutation.
A pSTAT5 in KSL cells

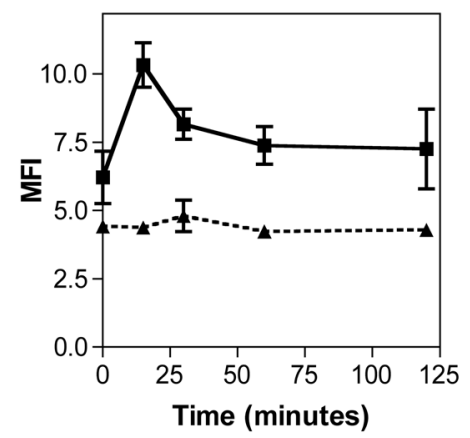

C

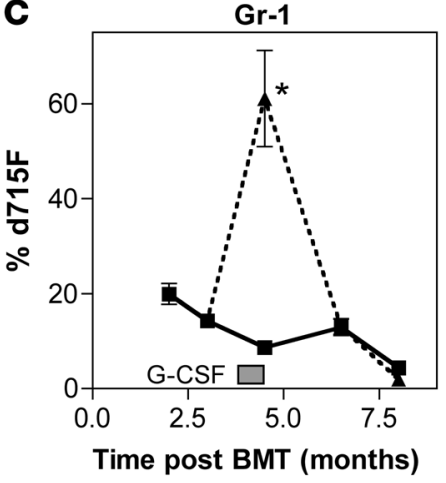

$\rightarrow-W T$

$-- \pm-d 715 F$

$\mathbf{B}$

B PSTAT3 in KSL cells

D

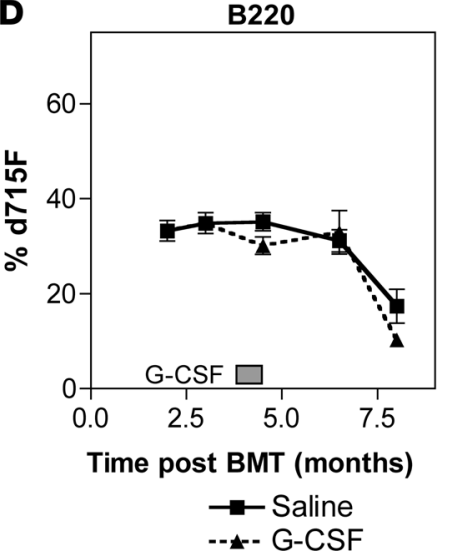

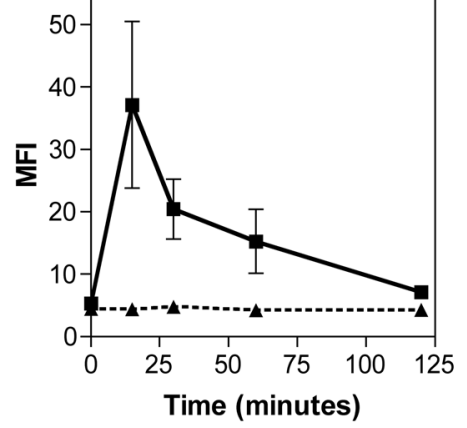

E

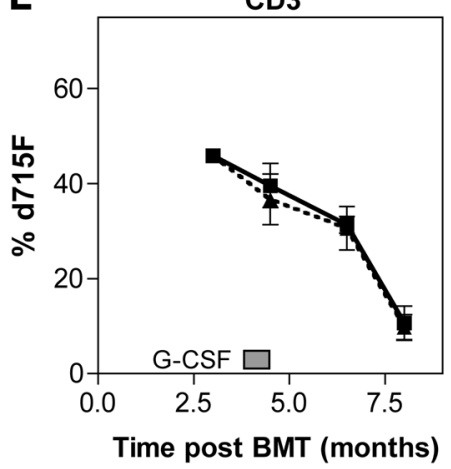

\section{Figure 7}

Competitive repopulation by HSC expressing the d715F G-CSFR is impaired. (A and B) Wild-type or d715F G-CSFR KSL cells were treated in vitro for the indicated times with G-CSF $(100 \mathrm{ng} / \mathrm{ml})$ and then incubated with antibodies specific for pStat5 (A) or pStat3 (B). The MFI of triplicate experiments is shown. (C-E) Irradiated syngeneic mice were reconstituted with a 1:1 ratio of d715F G-CSFR and wild-type bone marrow cells. The resulting chimeric mice were treated 4 months after bone marrow transplantation with saline alone or G-CSF $(10 \mu \mathrm{g} / \mathrm{kg} / \mathrm{d})$ for $21 \mathrm{days}$. The percentage of circulating neutrophils (C), B lymphocytes (D), and T lymphocytes (E) derived from d715F G-CSFR cells was determined at the indicated times ( $n=3-4$ for each group). Data represent the mean \pm SEM. ${ }^{*} P<0.05$ compared with saline-treated mice. 


\section{Methods}

Mice. d715 G-CSRR and d715F G-CSFR mice backcrossed 10 generations onto a C57BL/ 6 background were generated as described previously (15, 27). C57BL/6 mice (B6.SJL-Ptprc*/Pep3 ${ }^{\text {b }}$ BoyJ) that have the Ly5.1 gene were obtained from The Jackson Laboratory. Stat $5 \mathrm{a}^{+/-} \times$Stat $5 \mathrm{~b}^{+/-}$mice outbred on a NIH Black Swiss $\times 129 \mathrm{SvEv} \times \mathrm{C} 57 \mathrm{BL} / 6$ background were generated as previously described (59). These mice were intercrossed to generate Stat $5 \mathrm{a}^{-/-} \times$Stat $5 \mathrm{~b}^{-/-}$fetuses. Mice were housed in a specific pathogen-free environment. All experiments were approved by the Washington University Animal Studies Committee.

Competitive repopulation assay. Wild-type (Ly5.1), d715, or d715F G-CSFR (Ly5.2) bone marrow cells were harvested from strain- and sexmatched mice. A total of 5 million bone marrow cells were mixed at a 1:1 ratio and injected into the tail vein of lethally irradiated wild-type mice (Ly5.1). Recipient mice were conditioned with 1,000 cGy from a ${ }^{137}$ Cesium source at a rate of approximately $95 \mathrm{cGy} / \mathrm{min} 24$ hours prior to transplantation. Prophylactic antibiotics (trimethoprim-sulfamethoxazole; Alpharma) were given during the initial 2 weeks following transplantation. Mice were analyzed 3-12 months after transplantation. Where indicated, mice were treated with human recombinant G-CSF (Amgen) in PBS with $0.1 \%$ low-endotoxin BSA at a dose of $10 \mu \mathrm{g} / \mathrm{kg} / \mathrm{d}$ for 21 days. Secondary bone marrow transplantation was performed immediately following treatment with G-CSF or saline alone. Bone marrow cells $\left(5 \times 10^{6}\right)$ from the primary recipient mice were transplanted into secondary recipient mice.

Flow cytometry. Bone marrow and blood leukocytes were processed for flow cytometry as previously described (60). For chimerism analysis, cells were incubated with FITC-conjugated Ly5.1, Cy5-PE-conjugated Ly5.2, and one of the following PE-conjugated lineage markers: Gr-1 (myeloid), B220 (B lymphocytes), CD3 (T lymphocytes), or F4/80 (monocytes; Caltag Laboratories). Isotype-matched antibodies were used as negative controls. Cells were analyzed on a 2-laser, 5-color FACScan flow cytometer (BD Biosciences and Cytek Development). Unless otherwise indicated, all antibodies were obtained from BD Biosciences.

To analyze KSL cells, lineage ${ }^{+}$cells were first depleted. In brief, cells were incubated with the following PE-conjugated lineage markers: CD3, B220, Gr-1, and Ter-119. Following incubation with anti-PE colloid, lineage ${ }^{+}$cells were depleted using the AutoMacs system (Miltenyi Biotec). The lineagedepleted cells were then incubated with FITC-conjugated Sca-1, APC-conjugated c-kit, Cy5-PE-conjugated Ly5.2, and Cy7-APC-conjugated Ly5.1.

Cell cycle analysis of KSL cells. Following lineage depletion and incubation with FITC-conjugated Sca1 and APC-conjugated c-kit, bone marrow cells were incubated with $10 \mu \mathrm{g} / \mathrm{ml}$ Hoechst 33342 (Molecular Probes) in PBS containing $0.2 \% \mathrm{BSA}$ at $37^{\circ} \mathrm{C}$ for 60 minutes. Hoechst staining of the KSL cells was assessed using a MoFlo high-speed cell sorter (Dako).

BrdU labeling. Mice ( $n=5$ per condition) were injected with BrdU ( 2 doses of $1 \mathrm{mg}$ given 12 hours apart; $\mathrm{BD}$ Biosciences) and either saline alone of G-CSF ( 2 doses of $100 \mu \mathrm{g} / \mathrm{kg}$ given with the BrdU). Bone marrow cells were harvested 12 hours after the final dose of BrdU and incubated with FITC-conjugated CD48 (eBioscience) and CD41, biotinylated CD150 (clone number TC15-12F12.1; Biolegend), and the following panel of Cy5PE-conjugated lineage markers: CD3, B220, Gr-1, and Ter-119. Following incubation with streptavidin-PE (eBioscience), cells were fixed, permeabilized, and incubated with PE-conjugated anti-BrdU antibody per the manufacturer's recommendations (BrdU flow kit; BD Biosciences). Cells were analyzed using a MoFlo high-speed cell sorter.

Expression profiling and data analysis. Groups of 8-10 wild-type, d715 G-CSFR, or d715F G-CSFR mice were treated with a single subcutaneous injection of G-CSF $(100 \mu \mathrm{g} / \mathrm{kg})$ or saline alone. Bone marrow cells were harvested 3 hours later and KSL cells sorted. RNA was prepared from 20,000-100,000
KSL cells using Trizol reagent (Invitrogen). Total cellular RNA samples of $100 \mathrm{ng}$ were subjected to linear amplification, biotin labeling, fragmentation, and hybridization to Affymetrix MOE430 v2.0 GeneChip microarrays per protocols of the Siteman Cancer Center Multiplexed Gene Analysis Core Facility (http://pathology.wustl.edu/research/multiplexed.php). Experiments were performed in triplicate.

Visualization and statistical analysis of data were performed using Decision Site (Spotfire). Signal values corresponding to the probesets were normalized to adjust for minor differences in hybridization intensities across the 12 microarrays analyzed. After normalization, mean signal intensities for each probeset were calculated across the 3 experiments. Probesets upregulated or downregulated by G-CSF compared with saline control were selected based upon the following selection criteria: (a) present detection call in at least 2 of 3 probesets corresponding to the upregulated condition; (b) absolute signal intensity > 500 in at least 2 of 3 probesets corresponding to the upregulated condition; and (c) greater than 2-fold change in mean signal intensity (hereafter referred to as fold-induction ratio) between G-CSF and saline. In cases where multiple probesets corresponded to the same gene, the probeset with the highest average signal intensity and greatest number of present calls was used.

Assessment of Stat 5 and Stat 3 phosphorylation by flow cytometry. Bone marrow cells were either incubated with FITC-conjugated Gr-1 or lineage depleted and then incubated with FITC-Sca1 and APC-c-kit. Cells were then suspended in Opti-MEM (Invitrogen) with 10\% FBS and stimulated with G-CSF $(100 \mathrm{ng} / \mathrm{ml})$ for the indicated time at $37^{\circ} \mathrm{C}$. As a control, duplicate samples were cultured without G-CSF. Following incubation, cells were incubated in BD Phosphflow Lyse/fix buffer (BD Biosciences) for 10 minutes, permeabilized in $100 \%$ methanol at $4{ }^{\circ} \mathrm{C}$ for 20 minutes, and then incubated with anti-pStat5 (pY694) or anti-pStat3 (pY705) antibody (BD Biosciences) at room temperature for 30 minutes.

Retroviral transduction of Stat $5 a^{-1-} \times$ Stat $5 b^{-1-}$ fetal liver progenitors. Stable clones of the ecotropic packaging cell line GP+E86 containing the retroviral viral vector pMPncrdleno alone or this vector with cDNAs for wild-type murine G-CSFR or the d715 G-CSFR have been previously described (61). Cultures of fetal liver cells from 12- to 15-dpc Stat $5 \mathrm{a}^{-/-} \times$ Stat $5 b^{-/-}$fetuses were generated as previously described (62). Cells were cultured for 24 hours in RPMI containing $10 \% \mathrm{FBS}, 50 \mathrm{ng} / \mathrm{ml}$ of murine thrombopoietin, $100 \mathrm{ng} / \mathrm{ml}$ of murine stem cell factor, $10 \mathrm{ng} / \mathrm{ml}$ of murine interleukin-3, and $50 \mathrm{ng} / \mathrm{ml}$ of human flt 3 ligand at $37^{\circ} \mathrm{C}$ in $5 \% \mathrm{CO}_{2}$ (all cytokines were obtained from Peprotech). Cells were then cocultured in the same media with irradiated (1,500 cGy) GP+E86 cells for 48 hours. Viable cells $\left(0.5-1 \times 10^{5}\right.$ cells $)$ recovered from these cultures were plated in MethoCult M3231 (Stem Cell Technologies) supplemented with 1 or $10 \mathrm{ng} / \mathrm{ml}$ of human G-CSF and $1 \mathrm{mg} / \mathrm{ml}$ of geneticin (Invitrogen). Colonies containing at least 50 cells were scored on days 7-10 of culture. To determine transduction efficiency, $1-2 \times 10^{4}$ cultured fetal liver cells were placed in MethoCult M3434 with or without $1 \mathrm{mg} / \mathrm{ml}$ of geneticin and the percentage of colonies that were resistant to geneticin measured. A similar transduction efficiency (range 30\%-52\%) for all 3 retroviruses with both wild-type and $S t a 5 a^{-1-} \times$ Stat $5 b^{-/-}$cells was observed (data not shown).

Statistics. Statistical significance was determined either by a 2-way ANOVA analysis or by a 2 -sided Student $t$ test. All data represent the mean \pm SEM, unless otherwise stated.

\section{Acknowledgments}

We thank Amgen for their generous gift of G-CSF. We also thank Timothy A. Graubert for his critical review of this manuscript. This work was supported by NIH grant CA101937 and by the intramural program of the NIDDK/NIH. 
Received for publication May 16, 2007, and accepted in revised form December 19, 2007.
St. Louis, Missouri 63110, USA. Phone: (314) 362-8771; Fax: (314) 362-9333; E-mail: dlink@im.wustl.edu.

Address correspondence to: Daniel C. Link, Division of Oncology, Fulu Liu, Ghada Kunter, and Maxwell M. Krem contributed equalDepartment of Medicine, 660 S. Euclid Avenue, Campus Box 8007, ly to this work.

1. Bonnet, D., and Dick, J.E. 1997. Human acute myeloid leukemia is organized as a hierarchy that originates from a primitive hematopoietic cell. Nat. Med. 3:730-737.

2. Catlin, S.N., Guttorp, P., and Abkowitz, J.L. 2005. The kinetics of clonal dominance in myeloproliferative disorders. Blood. 106:2688-2692.

3. Jamieson, C.H., et al. 2004. Granulocyte-macrophage progenitors as candidate leukemic stem cells in blast-crisis CML. N. Engl. J. Med. 351:657-667.

4. Kustikova, O.S., et al. 2007. Retroviral vector insertion sites associated with dominant hematopoietic clones mark "stemness" pathways. Blood. 109:1897-1907.

5. Kostmann, R. 1956. Infantile genetic agranulocytosis: a new recessive lethal disease in man. Acta Paediatr. 105:1-78.

6. Welte, K., Zeidler, C., and Dale, D.C. 2006. Severe congenital neutropenia. Semin. Hematol. 43:189-195.

7. Donadieu, J., et al. 2005. Analysis of risk factors for myelodysplasias, leukemias and death from infection among patients with congenital neutropenia. Experience of the French Severe Chronic Neutropenia Study Group. Haematologica. 90:45-53.

8. Rosenberg, P.S., et al. 2006. The incidence of leukemia and mortality from sepsis in patients with severe congenital neutropenia receiving long-term G-CSF therapy. Blood. 107:4628-4635.

9. Dale, D.C., et al. 1993. A randomized controlled phase III trial of recombinant human granulocyte colony-stimulating factor (filgrastim) for treatment of severe chronic neutropenia. Blood. 81:2496-2502.

10. Germeshausen, M., Ballmaier, M., and Welte, K. 2007. Incidence of CSF3R mutations in severe congenital neutropenia and relevance for leukemogenesis: Results of a long-term survey. Blood. 109:93-99.

11. Dong, F., et al. 1995. Mutations in the gene for the granulocyte colony-stimulating-factor receptor in patients with acute myeloid leukemia preceded by severe congenital neutropenia. N. Engl. J. Med. 333:487-493.

12. Hermans, M.H., et al. 1999. Sustained receptor activation and hyperproliferation in response to granulocyte colony-stimulating factor (G-CSF) in mice with a severe congenital neutropenia/acute myeloid leukemia-derived mutation in the G- CSF receptor gene. J. Exp. Med. 189:683-692.

13. Hunter, M.G., and Avalos, B.R. 2000. Granulocyte colony-stimulating factor receptor mutations in severe congenital neutropenia transforming to acute myelogenous leukemia confer resistance to apoptosis and enhance cell survival. Blood. 95:2132-2137.

14. Hermans, M.H., et al. 1998. Perturbed granulopoiesis in mice with a targeted mutation in the granulocyte colony-stimulating factor receptor gene associated with severe chronic neutropenia. Blood. 92:32-39.

15. McLemore, M.L., Poursine-Laurent, J., and Link, D.C. 1998. Increased granulocyte colony-stimulating factor responsiveness but normal resting granulopoiesis in mice carrying a targeted granulocyte colony-stimulating factor receptor mutation derived from a patient with severe congenital neutropenia. J. Clin. Invest. 102:483-492.

16. Guba, S.C., Sartor, C.A., Hutchinson, R., Boxer, L.A., and Emerson, S.G. 1994. Granulocyte colonystimulating factor (G-CSF) production and G-CSF receptor structure in patients with congenital neu- tropenia. Blood. 83:1486-1492.

17. Kiel, M.J., et al. 2005. SLAM family receptors distinguish hematopoietic stem and progenitor cells and reveal endothelial niches for stem cells. Cell. 121:1109-1121.

18. Nozaki, I., et al. 2005. Small proline-rich proteins 2 are noncoordinately upregulated by IL-6/STAT3 signaling after bile duct ligation. Lab. Invest. 85:109-123.

19. Kelly, J.A., et al. 2003. Stat5 synergizes with T cell receptor/antigen stimulation in the development of lymphoblastic lymphoma. J. Exp. Med. 198:79-89.

20. Miccoli, M.C., Vaccarelli, G., Lanave, C., Cribiu, E.P., and Ciccarese, S. 2005. Comparative analyses of sheep and human TRG joining regions: evolution of $J$ genes in Bovidae is driven by sequence conservation in their promoters for germline transcription. Gene. 355:67-78.

21. Mizuki, M., et al. 2003. Suppression of myeloid transcription factors and induction of STAT response genes by AML-specific Flt3 mutations. Blood. 101:3164-3173.

22. Giraud, S., Hurlstone, A., Avril, S., and Coqueret, O. 2004. Implication of BRG1 and cdk9 in the STAT3mediated activation of the $\mathrm{p} 21 \mathrm{waf} 1$ gene. Oncogene. 23:7391-7398.

23. Takahashi, S., Harigae, H., Kaku, M., Sasaki, T., and Licht, J.D. 2004. Flt3 mutation activates p21WAF1/ CIP1 gene expression through the action of STAT5. Biochem. Biophys. Res. Commun. 316:85-92.

24. Mitchell, T.J., Whittaker, S.J., and John, S. 2003. Dysregulated expression of COOH-terminally truncated Stat 5 and loss of IL2-inducible Stat5dependent gene expression in Sezary Syndrome. Cancer Res. 63:9048-9054.

25. Krebs, D.L., and Hilton, D.J. 2001. SOCS proteins: negative regulators of cytokine signaling. Stem Cells. 19:378-387.

26. Irish, J.M., et al. 2004. Single cell profiling of potentiated phospho-protein networks in cancer cells. Cell. 118:217-228.

27. McLemore, M.L., et al. 2001. STAT-3 activation is required for normal G-CSF-dependent proliferation and granulocytic differentiation. Immunity. 14:193-204.

28. Yao, Z., et al. 2006. Stat5a/b are essential for normal lymphoid development and differentiation. Proc. Natl. Acad. Sci. U. S. A. 103:1000-1005.

29. Li, G., et al. 2007. STAT5 requires the $\mathrm{N}$-domain to maintain hematopoietic stem cell repopulating function and appropriate lymphoid-myeloid lineage output. Exp. Hematol. 35:1684.

30. Bernard, T., Gale, R.E., and Linch, D.C. 1996. Analysis of granulocyte colony stimulating factor receptor isoforms, polymorphisms and mutations in normal haemopoietic cells and acute myeloid leukaemia blasts. Br. J. Haematol. 93:527-533.

31. Carapeti, M., et al. 1997. Rarity of dominant-negative mutations of the G-CSF receptor in patients with blast crisis of chronic myeloid leukemia or de novo acute leukemia. Lenkemia. 11:1005-1008.

32. Glimm, H., Oh, I.H., and Eaves, C.J. 2000. Human hematopoietic stem cells stimulated to proliferate in vitro lose engraftment potential during their $\mathrm{S} / \mathrm{G}(2) / \mathrm{M}$ transit and do not reenter $\mathrm{G}(0)$. Blood. 96:4185-4193.

33. Passegue, E., Wagers, A.J., Giuriato, S., Anderson, W.C., and Weissman, I.L. 2005. Global analysis of proliferation and cell cycle gene expression in the regulation of hematopoietic stem and progenitor cell fates. J. Exp. Med. 202:1599-1611.
34. Cheng, T., et al. 2000. Hematopoietic stem cell quiescence maintained by 21 cip $1 /$ waf 1 . Science. 287:1804-1808.

35. Zhang, J., et al. 2006. PTEN maintains haematopoietic stem cells and acts in lineage choice and leukaemia prevention. Nature. 441:518-522.

36. Yilmaz, O.H., et al. 2006. Pten dependence distinguishes haematopoietic stem cells from leukaemiainitiating cells. Nature. 441:475-482.

37. Kato, Y., et al. 2005. Selective activation of STAT5 unveils its role in stem cell self-renewal in normal and leukemic hematopoiesis. J. Exp. Med. 202:169-179.

38. Zhang, S., et al. 2000. Essential role of signal transducer and activator of transcription (Stat)5a but not Stat5b for Flt3-dependent signaling. J. Exp. Med. 192:719-728.

39. Bunting, K.D., et al. 2002. Reduced lymphomyeloid repopulating activity from adult bone marrow and fetal liver of mice lacking expression of STAT5. Blood. 99:479-487.

40. Schuringa, J.J., Chung, K.Y., Morrone, G., and Moore, M.A.S. 2004. Constitutive activation of STAT5A promotes human hematopoietic stem cell self-renewal and erythroid differentiation. J. Exp. Med. 200:623-635.

41. Moriggl, R., et al. 2005. Stat5 tetramer formation is associated with leukemogenesis. Cancer Cell. 7:87-99.

42. Hayakawa, F., et al. 2000. Tandem-duplicated Flt3 constitutively activates STAT5 and MAP kinase and introduces autonomous cell growth in IL-3dependent cell lines. Oncogene. 19:624-631.

43. Schwaller, J., et al. 2000. Stat5 Is Essential for the Myelo- and Lymphoproliferative Disease Induced by TEL/JAK2. Mol. Cell. 6:693.

44. Hoelbl, A., et al. 2006. Clarifying the role of Stat5 in lymphoid development and Abelson-induced transformation. Blood. 107:4898-4906.

45. Schepers, H., et al. 2007. STAT5 is required for long-term maintenance of normal and leukemic human stem/progenitor cells. Blood. 110:2880.

46. Iwama, A., et al. 2004. Enhanced self-renewal of hematopoietic stem cells mediated by the polycomb gene product Bmi-1. Immunity. 21:843-851.

47. Antonchuk, J., Sauvageau, G., and Humphries, R.K. 2002. HOXB4-induced expansion of adult hematopoietic stem cells ex vivo. Cell. 109:39-45.

48. Lacorazza, H.D., et al. 2006. The transcription factor MEF/ELF4 regulates the quiescence of primitive hematopoietic cells. Cancer Cell. 9:175-187.

49. Arai, F., et al. 2004. Tie2/angiopoietin-1 signaling regulates hematopoietic stem cell quiescence in the bone marrow niche. Cell. 118:149-161.

50. Reya, T., et al. 2003. A role for Wnt signalling in self-renewal of haematopoietic stem cells. Nature. 423:409-414.

51. Zhang, P., et al. 2004. Enhancement of hematopoietic stem cell repopulating capacity and self-renew$\mathrm{al}$ in the absence of the transcription factor C/EBP alpha. Immunity. 21:853-863.

52. Yuan, Y., Shen, H., Franklin, D.S., Scadden, D.T., and Cheng, T. 2004. In vivo self-renewing divisions of haematopoietic stem cells are increased in the absence of the early G1-phase inhibitor, p18INK4C. Nat. Cell Biol. 6:436-442.

53. Duncan, A.W., et al. 2005. Integration of Notch and Wntsignaling in hematopoieticstem cell maintenance. Nat. Immunol. 6:314-322.

54. Hock, H., et al. 2004. Gfi-1 restricts proliferation and preserves functional integrity of haematopoietic stem cells. Nature. 431:1002-1007. 
55. Zeng, H., Yucel, R., Kosan, C., Klein-Hitpass, L., and Moroy, T. 2004. Transcription factor Gfi1 regulates self-renewal and engraftment of hematopoietic stem cells. EMBO J. 23:4116-4125.

56. Zhu, J., Zhang, Y., Joe, G.J., Pompetti, R., and Emerson, S.G. 2005. NF-Ya activates multiple hematopoietic stem cell (HSC) regulatory genes and promotes HSC self-renewal. Proc. Natl. Acad. Sci. U. S. A. 102:11728-11733.

57. Adam, M., et al. 2006. Targeting PIM kinases impairs survival of hematopoietic cells transformed by kinase inhibitor-sensitive and kinase inhibitor- resistant forms of Fms-like tyrosine kinase 3 and BCR/ABL. Cancer Res. 66:3828.

58. Jeha, S., et al. 2000. Spontaneous remission of granulocyte colony-stimulating factor-associated leuke$\mathrm{mia}$ in a child with severe congenital neutropenia. Blood. 96:3647-3649.

59. Cui, Y., et al. 2004. Inactivation of Stat5 in mouse mammary epithelium during pregnancy reveals distinct functions in cell proliferation, survival, and differentiation. Mol. Cell. Biol. 24:8037.

60. Richards, M.K., Liu, F., Iwasaki, H., Akashi, K., and Link, D.C. 2003. Pivotal role of granulocyte colo- ny-stimulating factor in the development of progenitors in the common myeloid pathway. Blood. 102:3562-3568

61. Jacob, J., Haug, J.S., Raptis, S., and Link, D.C. 1998. Specific signals generated by the cytoplasmic domain of the granulocyte colony-stimulating factor (G-CSF) receptor are not required for G-CSFdependent granulocytic differentiation. Blood. 92:353.

62. Cain, J.A., et al. 2007. Myeloproliferative disease induced by TEL-PDGFRB displays dynamic range sensitivity to Stat5 gene dosage. Blood. 109:3906. 